\title{
Elastic scattering of low-energy electrons by benzene
}

\author{
M. H. F. Bettega, ${ }^{\text {a) }}$ C. Winstead, and V. McKoy \\ A. A. Noyes Laboratory of Chemical Physics, California Institute of Technology, Pasadena, California 91125
}

(Received 2 December 1999; accepted 29 February 2000)

We present elastic cross sections obtained from ab initio calculations for low-energy electron scattering by benzene, $\mathrm{C}_{6} \mathrm{H}_{6}$. The calculations employed the Schwinger multichannel method as implemented for parallel computers within both the static-exchange and staticexchange-polarization approximations. We compare our results with other theoretical calculations and with available experimental data. In general, agreement is good. (C) 2000 American Institute of Physics. [S0021-9606(00)01120-X]

\section{INTRODUCTION}

Low-energy electron scattering by benzene has been the subject of recent theoretical and experimental studies. Sueoka ${ }^{1}$ and Mozejko et al. ${ }^{2}$ have measured total cross sections for a very broad energy range. Gianturco and Lucchese $^{3}$ have computed integral, differential, and partial (by symmetry) elastic cross sections using a parameter-free exact-static-exchange-plus-correlation-polarization potential. They have found several shape resonances: one in the $E_{2 u}$ representation, located at $1.82 \mathrm{eV}$; another in $B_{2 g}$, located at $7.44 \mathrm{eV}$; a third in $E_{1 u}$, located at $10.07 \mathrm{eV}$; and a fourth in $A_{2 g}$, located at $21.17 \mathrm{eV}$. Experiments have placed the first two resonances at $1.1 \mathrm{eV}$ and $4.8 \mathrm{eV}$, respectively. ${ }^{4-9}$ Gulley et al. ${ }^{10}$ have measured absolute total cross sections for electron scattering by benzene at very low energies, also finding a shape resonance at $1.1 \mathrm{eV}$ assigned to $E_{2 u}$. More recently, Gulley and Buckman ${ }^{11,12}$ have measured differential cross section at several energies. However, no fully ab initio study on elastic scattering of electrons by benzene has yet been done.

$A b$ initio calculation of low-energy electron-molecule collision cross sections is computationally challenging. Even at the lowest level of approximation, the static-exchange (SE) approximation, in which only direct (Coulomb) and exchange interactions between the incident electron and the molecule are considered, the computational demands grow severe for larger molecules. Pseudopotentials have proven effective in dealing with molecules containing heavier atoms, ${ }^{13}$ both within the static-exchange approximation ${ }^{14}$ and also in more complex calculations that include simultaneously multichannel coupling and polarization effects. ${ }^{15}$ Massively parallel computers have also been used with success to obtain elastic and inelastic cross sections for scattering of electrons by large molecules. ${ }^{16}$

In this work, we report results from a completely $a b$ initio calculation of elastic scattering of low-energy electrons by benzene. Benzene is an intrinsically interesting target molecule due to its geometry and symmetry and is of additional interest in light of recent experimental and theoretical

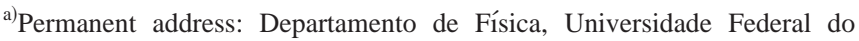
Paraná, Caixa Postal 19081, 81531-990, Curitiba, Paraná, Brazil.
}

studies. ${ }^{1-3,10-12}$ Our calculations employed the Schwinger multichannel (SMC) method ${ }^{17,18}$ as implemented for parallel computers ${ }^{19}$ and were carried out both in the static-exchange and in the static-exchange-plus-polarization (SEP) approximations. This study may be considered a first step toward studies of more complex aromatic molecules such as the fluoro-, chloro-, and fluorochlorobenzenes ${ }^{20}$ or the DNA bases. $^{21}$

\section{COMPUTATIONAL PROCEDURES}

The SMC method has been described in detail. ${ }^{17,18} \mathrm{We}$ do not repeat that description here but rather focus on the details of the present calculations. Both our SE and SEP calculations used the fixed-nuclei approximation at the experimental $D_{6 h}$ geometry, $r(\mathrm{C}-\mathrm{C})=1.397 \AA$ and $r(\mathrm{C}-\mathrm{H})$ $=1.084 \AA .^{22}$ To describe the occupied and scattering orbitals we used the $6-311++\mathrm{G}(2 d, p)$ basis set internal to the electronic structure program GAMESS, ${ }^{23}$ which includes all six combinations of Cartesian $d$-type functions. It has been noted that the symmetric " $3 s$ " combination, namely $\left[\left(x^{2}+y^{2}\right.\right.$ $\left.\left.+z^{2}\right) \exp \left(-\alpha r^{2}\right)\right]$, may, in combination with the diffuse $s$-type Gaussians, give rise to linear dependence in the basis set and thereby to spurious resonances in the cross sections. ${ }^{14}$ Accordingly, we have modified the exponents of the diffuse $s$ - and $p$-type functions, as well as the exponents of the $d$-type functions, to minimize linear dependence. The final exponents chosen are shown in Table I.

In our SE calculations, we used the canonical HartreeFock (HF) virtual orbitals as scattering orbitals. The number of virtual orbitals belonging to each irreducible representation of $D_{6 h}$ appears in Table II as the number of SE configurations for that representation. Each component of the twofold degenerate $E$ representations is computed separately, giving a total of 16 independent contributions to the cross section. Because the components of the $E$ representations are not equivalent with respect to the numerical quadratures we used, the mutual agreement of these components allows us to assess the convergence of the quadratures. In fact, we found that the degenerate components of the $E$ representations agreed to within $2 \%$ or better at every energy.

To include polarization effects in the SEP calculations, we followed, with some modification, the procedure used 
TABLE I. Original (Ref. 23) and modified diffuse $s$, diffuse $p$, and $d$ exponents for carbon used in $e^{-}-\mathrm{C}_{6} \mathrm{H}_{6}$ collision calculations.

\begin{tabular}{ccc}
\hline \hline Type & Original & Modified \\
\hline$s$ & 0.0438 & 0.06 \\
$p$ & 0.0438 & 0.06 \\
$d$ & 1.252 & 1.60 \\
$d$ & 0.313 & 0.40 \\
\hline \hline
\end{tabular}

successfully in previous studies of electron scattering by $\mathrm{N}_{2} \mathrm{O}$ (Ref. 24) and $\mathrm{CO}_{2}{ }^{25}$ In the $\mathrm{N}_{2} \mathrm{O}$ and $\mathrm{CO}_{2}$ studies, we used one approach for resonant irreducible representations and another, more computationally demanding, approach only in the totally symmetric representation. For $\mathrm{C}_{6} \mathrm{H}_{6}$, we found that it was necessary to apply the more demanding approach not only in the totally symmetric $A_{1 g}$ representation but also in any other representation where the background cross section (i.e., the cross section due to direct, nonresonant scattering) was substantial at low collision energies. Thus the procedure followed in the present calculations was as follows:

In irreducible representations where shape resonances occur but where the background cross section is small, we constructed a valencelike "modified virtual orbital" (MVO) $\widetilde{\varphi}$ by diagonalizing a cationic Fock operator within the space of virtual orbitals of the neutral. ${ }^{26}$ To build up the $(N+1)$-particle configuration space, we considered only single excitations that preserved the ground state symmetry, i.e., symmetry- and spin-preserving excitations of the type $\left[p_{\gamma} \rightarrow q_{\gamma}\right] \widetilde{\varphi}$, where $p_{\gamma}$ is an occupied and $q_{\gamma}$ a virtual orbital, both belonging to the same representation $\gamma$. The target and the temporary-anion wave functions were thus described in a balanced way, and overcorrelation of the anion was avoided. For nonresonant symmetries and for resonant symmetries with a significant background cross section, we built up a compact set of polarizing orbitals from the occupied and virtual orbitals and from canonical orbital energies. ${ }^{27}$ These polarizing orbitals are defined by

$$
\varphi_{i, \mu}=\sum_{j \in \text { virtuals }} \frac{\left\langle\varphi_{j}\left|x_{\mu}\right| \varphi_{i}\right\rangle}{E_{j}-E_{i}} \varphi_{j},
$$

where $\varphi_{i}$ is an occupied orbital, $x_{\mu}$ is a component of the dipole operator, and the sum runs over the HF virtual orbitals. The HF energies $E_{j}$ were shifted downward by a small constant in recognition of the fact that they are computed in the presence of an $N$-electron, rather than $(N-1)$-electron, core. The Schmidt procedure was used to construct an orthonormal set from the polarizing orbitals and the residual scattering orbitals. Polarization was taken into account by allow-
TABLE III. Partial-wave decomposition of the $D_{6 h}$ group.

\begin{tabular}{ccc}
\hline \hline Symmetry & $l$ & $m(\bmod 6)$ \\
\hline$a_{1 g}$ & 0 & 0 \\
$a_{1 u}$ & 7 & 6 \\
$a_{2 g}$ & 6 & 6 \\
$a_{2 u}$ & 1 & 0 \\
$b_{1 g}$ & 4 & 3 \\
$b_{1 u}$ & 3 & 3 \\
$b_{2 g}$ & 4 & 3 \\
$b_{2 u}$ & 3 & 3 \\
$e_{1 g}$ & & 1,5 \\
$e_{1 u}$ & & 1,5 \\
$e_{2 g}$ & & 2,4 \\
$e_{2 u}$ & & 2,4 \\
\hline \hline
\end{tabular}

ing single excitations from occupied valence orbitals into this set of polarizing orbitals, while employing the entire set of virtual orbitals as scattering orbitals.

More specifically, for each of the resonant representations where direct scattering is weak, namely, $E_{2 u}, B_{2 g}$, and $A_{2 g}$, we constructed a valencelike MVO from a +4 cation Fock operator, +4 being the minimum charge necessary to obtain a totally symmetric closed-shell cation wave function given that the highest occupied orbital of the neutral is twofold degenerate. We included only single excitations out of the 15 valence orbitals in constructing the $(N+1)$-particle configuration space. The number of configurations obtained with this procedure is shown in Table II. For the two-fold degenerate representations, $E_{2 g}$ and $E_{1 u}$, we polarized only one component. The other component was obtained by rotation of the scattering amplitude after first expanding it in partial waves. In performing all partial-wave decompositions, we expanded the scattering amplitude up to $l=10$. For the $A_{1 g}, E_{2 g}, E_{1 u}$, and $A_{2 u}$ representations, we included a substantial number of configurations using a compact set of polarizing orbitals as described above. For these symmetries, we worked in the $D_{2 h}$ subgroup and then recovered the corresponding $D_{6 h}$ contributions from the partial-wave decomposition of the scattering amplitude, according to Table III. ${ }^{28}$ Table IV presents the relation between the representations of the $D_{2 h}$ and $D_{6 h}$ groups. The five remaining irreducible representations, namely $A_{1 u}, B_{1 g}, B_{1 u}, B_{2 u}$, and $E_{1 g}$, were included through the static-exchange approximation.

\section{RESULTS}

Figure 1 shows our SE and SEP integral elastic cross sections (ICS) along with the SEP results of Gianturco and Lucchese $^{3}$ and the measured total scattering cross sections of

TABLE II. Number of configurations used in $e^{-}-\mathrm{C}_{6} \mathrm{H}_{6}$ collision calculations in the static-exchange (SE) and static-exchange-polarization (SEP) approximations.

\begin{tabular}{lrrrrrrrrrrrrr}
\hline \hline & $a_{1 g}^{a}$ & $a_{2 g}$ & $b_{1 g}$ & $b_{2 g}$ & $e_{1 g}$ & $e_{2 g}$ & $a_{1 u}$ & $a_{2 u}^{a}$ & $b_{1 u}$ & $b_{2 u}$ & $e_{1 u}^{a}$ & $e_{2 u}$ & Total \\
\hline SE & 17 & 2 & 2 & 7 & 16 & 48 & 2 & 6 & 18 & 6 & 48 & 18 & 195 \\
SEP & 2799 & 279 & 2 & 279 & 16 & 48 & 2 & 2000 & 18 & 6 & 2777 & 562 & 8788 \\
\hline \hline
\end{tabular}

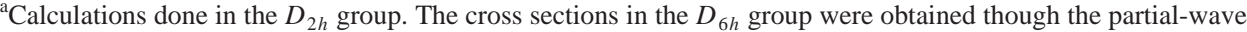
decomposition of the scattering amplitude. 
TABLE IV. Relation between the representations of $D_{2 h}$ and $D_{6 h}$ groups.

\begin{tabular}{cc}
\hline \hline$D_{2 h}$ & $D_{6 h}$ \\
\hline$a_{g}$ & $a_{1 g}+e_{2 g}$ \\
$b_{1 g}$ & $b_{1 g}+e_{1 g}$ \\
$b_{2 g}$ & $b_{2 g}+e_{1 g}$ \\
$b_{3 g}$ & $a_{2 g}+e_{2 g}$ \\
$a_{u}$ & $a_{1 u}+e_{2 u}$ \\
$b_{1 u}$ & $b_{1 u}+e_{1 u}$ \\
$b_{2 u}$ & $b_{2 u}+e_{1 u}$ \\
$b_{3 u}$ & $a_{2 u}+e_{2 u}$ \\
\hline \hline
\end{tabular}

Sueoka ${ }^{1}$ and of Mozejko et al. ${ }^{2}$ Our SEP results are in generally good agreement with those of Gianturco and Lucchese, except at lower energies. Part of the difference may arise because, as discussed above, we obtained the contributions of certain irreducible representations within the staticexchange approximation; however, as described below, most of the disagreement is due to a few representations for which both calculations included polarization.

Figure 2 shows results for the three resonant representations associated with unoccupied valence molecular orbitals, namely, $E_{2 u}, B_{2 g}$, and $A_{2 g}$. We also show in Fig. 2 our SE results and the SEP results of Gianturco and Lucchese. The spurious oscillations that appear in our cross sections at higher energies arise from treating as closed excitation channels that are in fact open at those energies. Such pseudoresonances are a common feature of polarization treatments of this type. As expected, including polarization moves the resonances from their static-exchange positions to lower energies: from $3.11 \mathrm{eV}$ to $2.3 \mathrm{eV}$ for the $E_{2 u}$ resonance, from $9.4 \mathrm{eV}$ to $8.3 \mathrm{eV}$ for $B_{2 g}$, and from $23 \mathrm{eV}$ to $21.8 \mathrm{eV}$ for $A_{2 g}$. The resonances positions obtained by Gianturco and Lucchese are uniformly lower: $1.82 \mathrm{eV}, 7.44 \mathrm{eV}$, and 21.17 $\mathrm{eV}$, respectively. Both of the calculated positions for the $B_{2 g}$ are too high compared to the experimental value, $4.8 \mathrm{eV}$. The size of the discrepancy is surprising and has no obvious origin, though we will discuss a tentative explanation below.

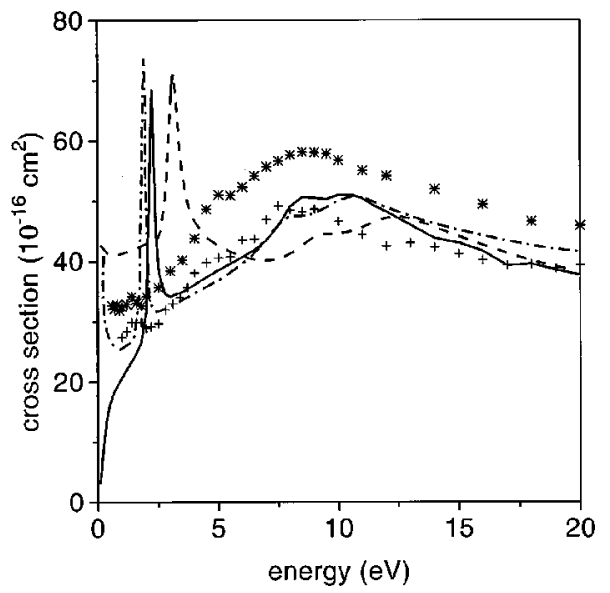

FIG. 1. Integral elastic electron-scattering cross section for $\mathrm{C}_{6} \mathrm{H}_{6}$. Dashed line, present static-exchange results; solid line, present results including polarization; dot-dashed line, results of Ref. 3; crosses, total cross section data of Ref. 1; stars, total cross section data of Ref. 2.

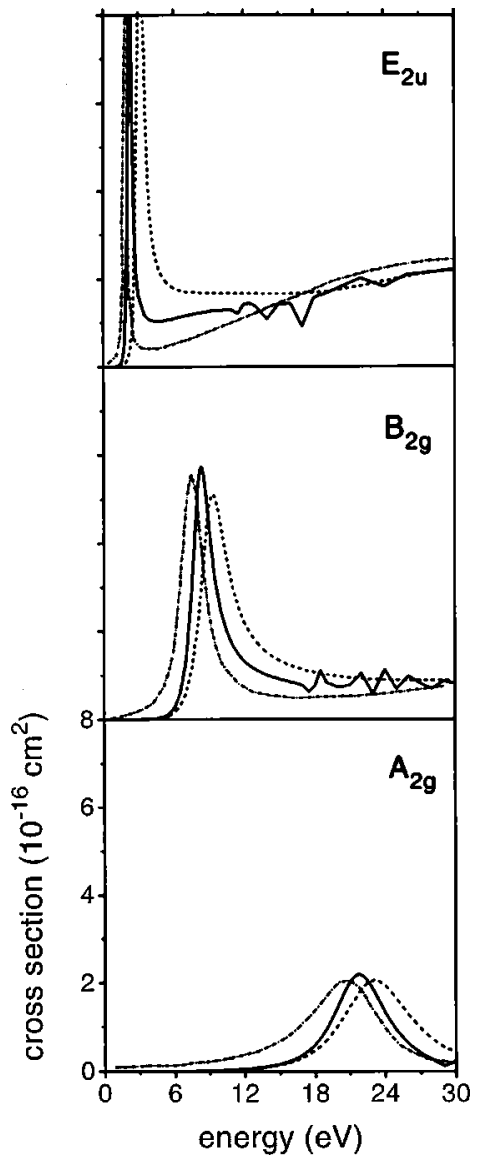

FIG. 2. Partial cross section for $E_{2 u}, B_{2 g}$, and $A_{2 g}$. Dashed line, present static-exchange results; solid line, present results including polarization; dotdashed line, results of Ref. 3.

In Fig. 3, we show results for the other representations for which we have included polarization, namely, $A_{1 g}, A_{2 u}$, $E_{1 u}$, and $E_{2 g}$. Our results mostly show good agreement with those of Gianturco and Lucchese. However, at very low energies, the $E_{1 u}, E_{2 g}$, and $A_{2 u}$ cross sections of Gianturco and Lucchese show a minimum and then rise to a finite value at $0 \mathrm{eV}$. For each of these representations, our cross sections vanish at $0 \mathrm{eV}$. Together, three representations account for much of the disagreement in the summed ICS that was seen in Fig. 1.

As Table IV shows, the $A_{g}$ representation of the $D_{2 h}$ subgroup results from mixing of the $A_{1 g}$ representation of $D_{6 h}$ with one component of the $E_{2 g}$ representation. As described above, partial-wave decomposition of the $A_{g}$ representation according to Table III was used to obtain the $A_{1 g}$ and $E_{2 g}$ cross sections shown in Fig. 3. As may be seen, the $E_{2 g}$ cross section exhibits considerable oscillation above 12 $\mathrm{eV}$. This structure does not appear to be due to numerical instability because the $A_{1 g}$ cross section is free of oscillations in this energy range. We thus attribute it to effects of closed channels that should be open at these energies. To avoid introducing this structure into the final results, we chose to obtain our integral and differential cross sections by including this representation within the SE approximation.

In Fig. 4, we show our SE results for the $E_{1 g}$ and $B_{1 u}$ irreducible representations. The cross sections for the $B_{2 u}$, 


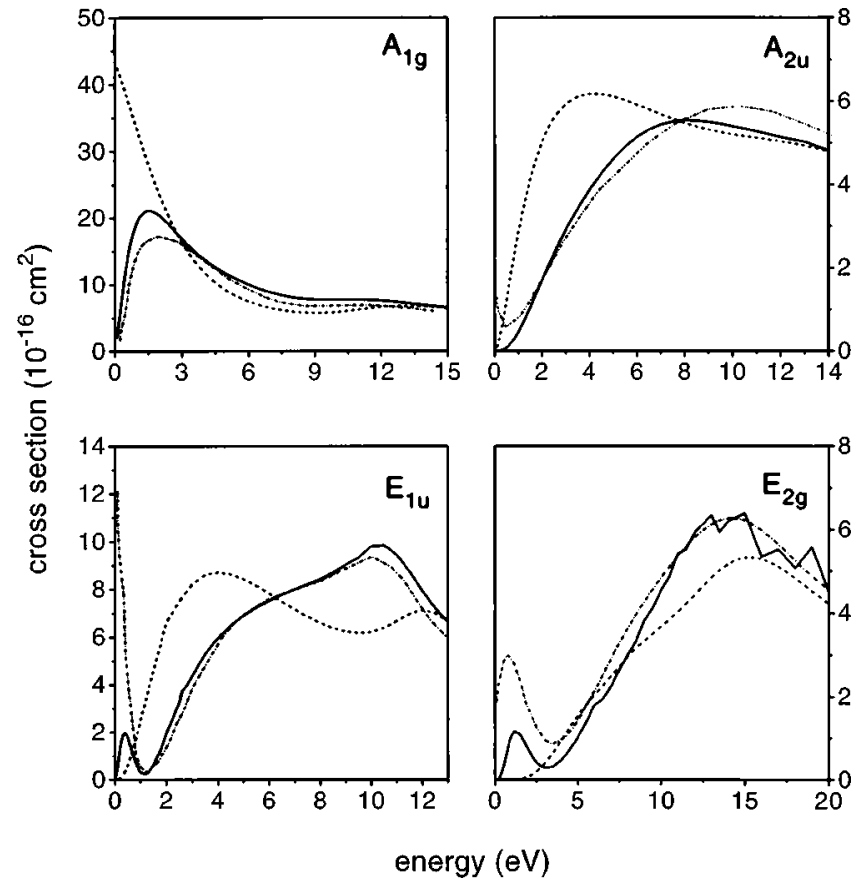

FIG. 3. Partial cross sections for $A_{1 g}, E_{1 u}, A_{2 u}$, and $E_{2 g}$. Dashed line, present static-exchange results; solid line, present results including polarization; dot-dashed line, results of Ref. 3.

$A_{1 u}$, and $B_{1 g}$ representations, also computed at the SE level, are small and not shown.

The differential cross sections (DCS) at the peak of the resonances in $E_{2 u}, B_{2 g}, A_{2 g}$, and $E_{1 u}(2.23,8.3,21.8$, and $10 \mathrm{eV}$, respectively) are shown in Fig. 5. The SEP results are shown, except at $21.8 \mathrm{eV}$, where we present our SE result. We also show the results of Gianturco and

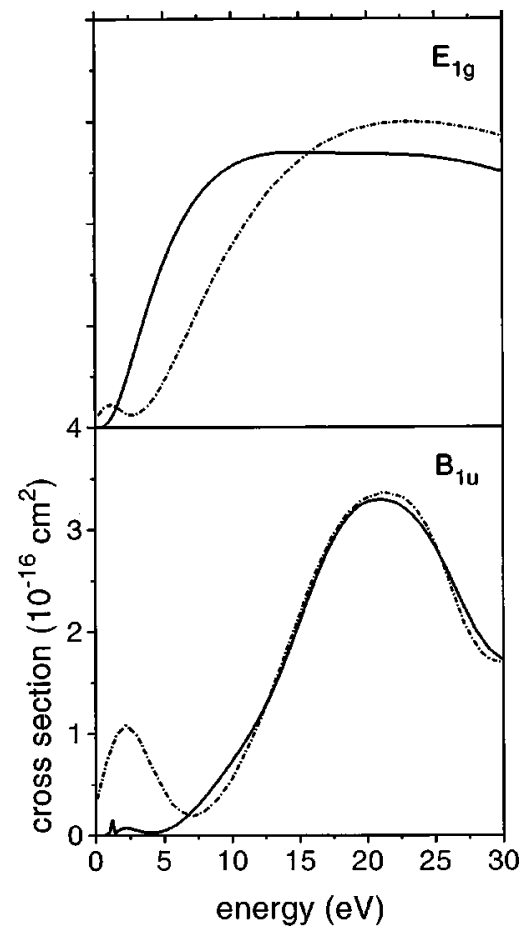

FIG. 4. Partial cross sections for $E_{1 g}$ and $B_{1 u}$. Solid line, present staticexchange results; dot-dashed line, results of Ref. 3.

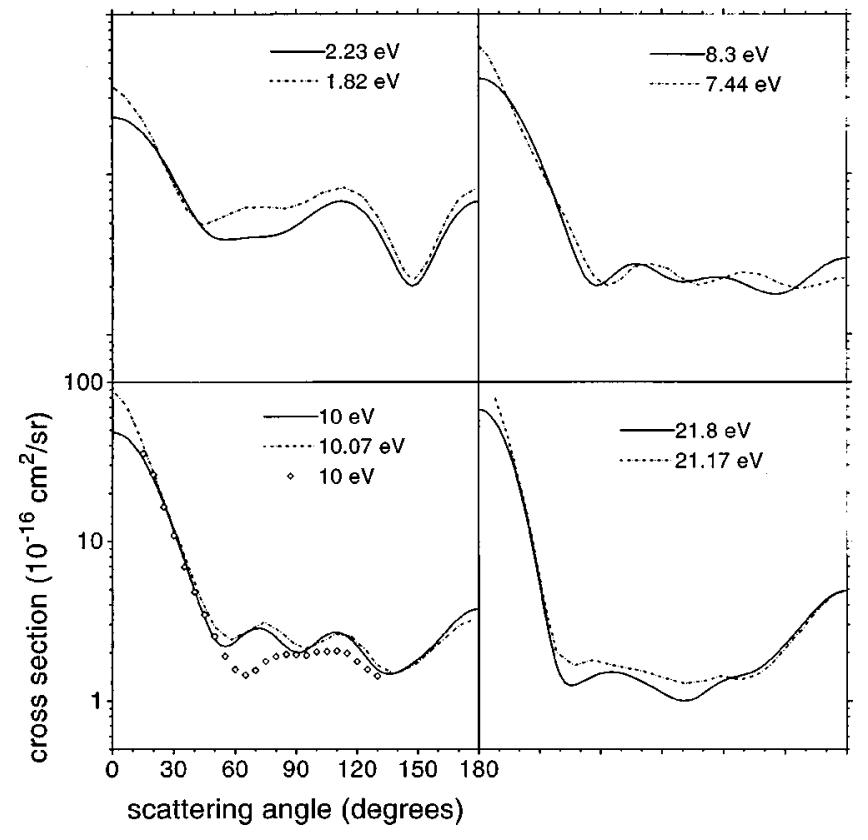

FIG. 5. Differential cross section at the peaks of the resonances of Fig. 2 and at the peak of the $E_{1 u}$ resonance. Solid line, present results; dot-dashed line, results of Ref. 3; diamonds, experimental data of Ref. 12.

Lucchese at $1.82 \mathrm{eV}\left(E_{2 u}\right), 7.44 \mathrm{eV}\left(B_{2 g}\right), 10.07 \mathrm{eV}\left(E_{1 u}\right)$, and $21.17 \mathrm{eV}\left(A_{2 g}\right)$, as well as the very recent measurements of Gulley and Buckman at $10 \mathrm{eV} .{ }^{12}$ Our DCS agree very well with the results of Gianturco and Lucchese at these energies, except at scattering angles below $20^{\circ}$, where we obtain smaller cross sections. At larger scattering angles, both calculations show the same structures. At $10 \mathrm{eV}$, we obtain good agreement with measurements of Gulley and Buckman between $20^{\circ}$ and $50^{\circ}, 1^{12}$ for larger scattering angles, the experimental and theoretical results show similar structures but differ in detail, especially between $50^{\circ}$ and $90^{\circ}$.

In Fig. 6, we show our DCS at 8.5, 15, 20, and $30 \mathrm{eV}$ along with the experimental data of Gulley and Buckman. ${ }^{11,12}$ The DCS at 15, 20, and $30 \mathrm{eV}$, where polarization is not very important, are SE results. Again, we find good agreement with results of Gulley and Buckman at small scattering angles. At $8.5 \mathrm{eV}$, our DCS exhibits minima near $40^{\circ}, 100^{\circ}$, and $140^{\circ}$. Corresponding but smaller undulations are visible in the DCS of Gulley and Buckman, the first minimum occurring near $70^{\circ}$ rather than $40^{\circ}$. At $20 \mathrm{eV}$, our DCS shows minima near $50^{\circ}$ and $100^{\circ}$. The measured DCS follows the same general shape. At $15 \mathrm{eV}$, we find similar qualitative agreement between our results and those of Gulley and Buckman for larger scattering angles. At $30 \mathrm{eV}$, the agreement is less good. At all four energies shown in Fig. 6, and also at $10 \mathrm{eV}$, Fig. 5, the experimental DCS is smaller than the theoretical DCS at intermediate scattering angles despite being in good quantitative agreement at smaller angles. Table V presents numerical values of our DCS at selected energies.

\section{DISCUSSION}

Our ICS with and without polarization, as well as the ICS of Gianturco and Lucchese, exhibit two broad maxima 


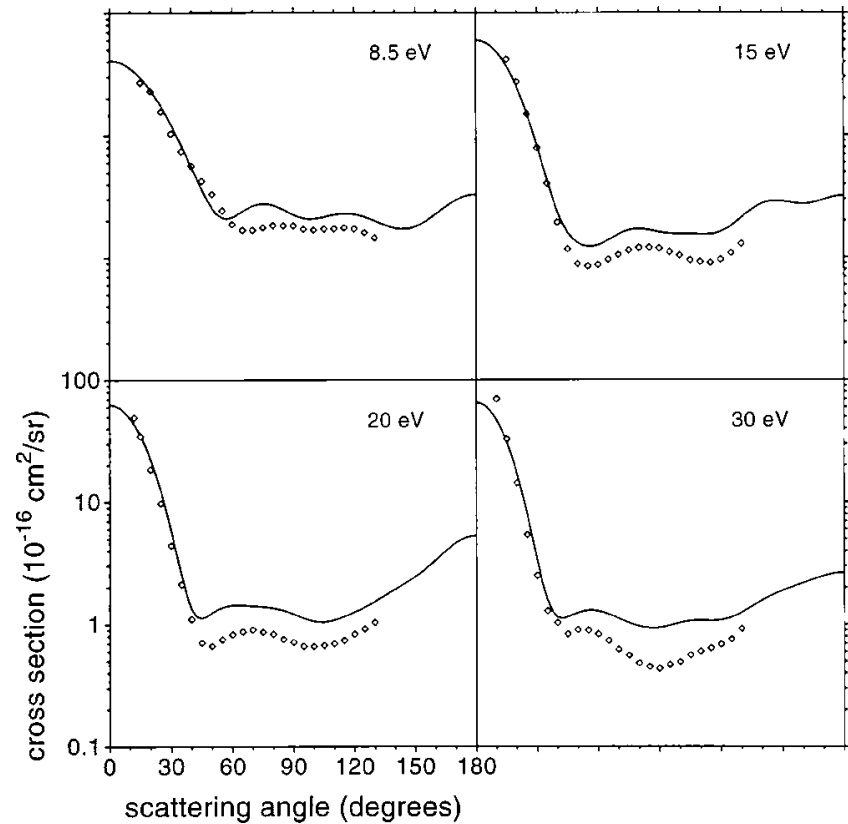

FIG. 6. Differential cross sections for $\mathrm{C}_{6} \mathrm{H}_{6}$ at $8.5,15,20$, and $30 \mathrm{eV}$. Solid line, present results; diamonds, experimental data of Refs. 11, 12.

between $8 \mathrm{eV}$ and $12 \mathrm{eV}$, as seen in Fig. 1. In our SE cross section and in the cross section of Gianturco and Lucchese, the second structure is larger in magnitude than the first, while in our SEP cross section the maxima are of approximately equal magnitude. As may be seen in Fig. 3, the difference arises from the $E_{2 g}$ representation. With the inclusion of polarization effects, the cross section increases by about $3 \AA^{2}$ (taking into account the two-fold degeneracy) in this energy range, making the second maximum in the ICS more prominent. We could have included the SEP result for $E_{2 g}$ in the summed SEP ICS at the expense of a more noisy cross section but, as discussed earlier, chose to employ the smooth SE result. Another minor source of error in our results could be the $E_{1 g}$ representation, which we also treated within the SE approximation. As shown in Fig. 4, there is a small difference between our SE results and the SEP results of Gianturco and Lucchese, which is again magnified by the two-fold degeneracy.

As discussed in the previous section and shown in Fig. 2, our calculated energy for the $B_{2 g}$ resonance is too high when compared with the experimental resonance position. Gianturco and Lucchese obtain a resonance energy that is somewhat lower but still too high compared to experiment. Since the calculations include, by design, all effects generally thought significant in determining the resonance energy, the large discrepancies between theory and experiment are surprising. A tentative explanation may be based on observations by Nenner and Schulz, ${ }^{7}$ Azria and Schulz, ${ }^{8}$ and more recently Allan. ${ }^{9}$ Azria and Schulz $^{8}$ proposed that this resonance decays not only into the ${ }^{1} A_{1 g}$ ground state of benzene but also into the three low-lying excited states, ${ }^{3} B_{1 u},{ }^{3} E_{1 u}$, and ${ }^{1} B_{2 u}$. In fact, Allan has observed that the $B_{2 g}$ shape resonance plays an important role in the excitation of the ${ }^{3} B_{1 u}$ and ${ }^{3} E_{1 u}$ states. He interpreted this result as experimental confirmation of configuration interaction between the $B_{2 g}$
TABLE V. Differential cross sections for $\mathrm{C}_{6} \mathrm{H}_{6}$ at selected energies (cross section in units of $10^{-16} \mathrm{~cm}^{2}$, scattering angle in degrees).

\begin{tabular}{rrrrrrrrr}
\hline \hline Angle & $2.23 \mathrm{eV}$ & $8.3 \mathrm{eV}$ & $8.5 \mathrm{eV}$ & $10 \mathrm{eV}$ & $15 \mathrm{eV}$ & $20 \mathrm{eV}$ & $21.8 \mathrm{eV}$ & $30 \mathrm{eV}$ \\
\hline 0 & 22.80 & 39.63 & 40.99 & 48.34 & 59.40 & 62.87 & 65.55 & 65.23 \\
5 & 22.21 & 38.25 & 39.53 & 46.28 & 56.29 & 59.05 & 61.27 & 60.09 \\
10 & 20.56 & 34.42 & 35.50 & 40.67 & 47.86 & 48.85 & 49.97 & 46.91 \\
15 & 18.07 & 28.98 & 29.77 & 32.91 & 36.43 & 35.47 & 35.42 & 30.89 \\
20 & 15.11 & 22.94 & 23.42 & 24.69 & 24.76 & 22.45 & 21.69 & 17.08 \\
25 & 12.07 & 17.20 & 17.41 & 17.38 & 15.02 & 12.30 & 11.43 & 7.99 \\
30 & 9.30 & 12.32 & 12.32 & 11.66 & 8.23 & 5.84 & 5.27 & 3.36 \\
35 & 7.06 & 8.48 & 8.37 & 7.61 & 4.28 & 2.56 & 2.34 & 1.58 \\
40 & 5.46 & 5.67 & 5.52 & 4.93 & 2.36 & 1.34 & 1.33 & 1.14 \\
45 & 4.49 & 3.75 & 3.63 & 3.29 & 1.59 & 1.12 & 1.19 & 1.15 \\
50 & 4.02 & 2.59 & 2.55 & 2.43 & 1.31 & 1.24 & 1.30 & 1.24 \\
55 & 3.89 & 2.06 & 2.11 & 2.18 & 1.23 & 1.37 & 1.42 & 1.29 \\
60 & 3.93 & 2.02 & 2.13 & 2.33 & 1.25 & 1.43 & 1.48 & 1.28 \\
65 & 4.00 & 2.24 & 2.38 & 2.64 & 1.37 & 1.43 & 1.47 & 1.21 \\
70 & 4.04 & 2.53 & 2.64 & 2.83 & 1.53 & 1.41 & 1.42 & 1.11 \\
75 & 4.08 & 2.70 & 2.76 & 2.77 & 1.66 & 1.39 & 1.37 & 1.03 \\
80 & 4.17 & 2.70 & 2.68 & 2.49 & 1.70 & 1.36 & 1.31 & 0.96 \\
85 & 4.38 & 2.54 & 2.46 & 2.17 & 1.67 & 1.30 & 1.25 & 0.93 \\
90 & 4.75 & 2.33 & 2.23 & 2.00 & 1.61 & 1.21 & 1.17 & 0.94 \\
95 & 5.27 & 2.17 & 2.09 & 2.07 & 1.56 & 1.12 & 1.08 & 0.98 \\
100 & 5.86 & 2.11 & 2.07 & 2.32 & 1.55 & 1.06 & 1.02 & 1.03 \\
105 & 6.38 & 2.13 & 2.14 & 2.58 & 1.55 & 1.05 & 1.03 & 1.07 \\
110 & 6.70 & 2.20 & 2.24 & 2.67 & 1.54 & 1.09 & 1.10 & 1.08 \\
115 & 6.67 & 2.25 & 2.29 & 2.52 & 1.55 & 1.16 & 1.20 & 1.07 \\
120 & 6.26 & 2.25 & 2.25 & 2.19 & 1.62 & 1.26 & 1.28 & 1.09 \\
125 & 5.48 & 2.17 & 2.14 & 1.83 & 1.81 & 1.38 & 1.36 & 1.14 \\
130 & 4.46 & 2.05 & 1.99 & 1.57 & 2.11 & 1.53 & 1.43 & 1.25 \\
135 & 3.41 & 1.92 & 1.84 & 1.47 & 2.46 & 1.72 & 1.55 & 1.40 \\
140 & 2.54 & 1.82 & 1.73 & 1.51 & 2.75 & 1.93 & 1.71 & 1.57 \\
145 & 2.05 & 1.77 & 1.70 & 1.63 & 2.89 & 2.17 & 1.94 & 1.74 \\
150 & 2.06 & 1.81 & 1.77 & 1.81 & 2.88 & 2.45 & 2.24 & 1.89 \\
155 & 2.58 & 1.95 & 1.96 & 2.05 & 2.79 & 2.82 & 2.65 & 2.03 \\
160 & 3.51 & 2.16 & 2.24 & 2.38 & 2.73 & 3.34 & 3.18 & 2.17 \\
165 & 4.63 & 2.44 & 2.59 & 2.80 & 2.79 & 3.97 & 3.81 & 2.32 \\
170 & 5.68 & 2.70 & 2.92 & 3.25 & 2.95 & 4.63 & 4.45 & 2.48 \\
175 & 6.44 & 2.90 & 3.17 & 3.60 & 3.12 & 5.12 & 4.94 & 2.59 \\
180 & 6.71 & 2.97 & 3.26 & 3.74 & 3.19 & 5.31 & 5.12 & 2.64 \\
\hline \hline & & & & & & & &
\end{tabular}

shape resonance and a core-excited $B_{2 g}$ resonance. While the formation of a shape resonance is a single-particle process in which the scattered electron temporarily occupies an empty molecular orbital (in this case of $b_{2 g}$ type), a core-excited shape resonance arises from a two-particle process involving excitation of the molecule with simultaneous capture of the scattered electron. Although both calculations go beyond a single-particle description-by including virtual excitations explicitly, in the present work, and via an effective potential in the work of Gianturco and Lucchese-they are primarily designed to capture the distortion of the target charge density in response to the presence of the projectile. In particular, the $(N+1)$-particle configuration space we used to calculate SEP results for $B_{2 g}$ was not chosen to give a good description of possible core-excited $B_{2 g}$ shape resonances built on the low-lying excited states of benzene. Further study of this interesting issue is certainly warranted.

The $E_{2 u}$ shape resonance occurs below the first electronic excitation threshold, and Allan therefore argues that configuration mixing of the above type should not occur. ${ }^{9}$ Nonetheless, the position of the $E_{2 u}$ resonance, though improved by inclusion of polarization effects, remains above 
the experimental position, which is about $1.1 \mathrm{eV}$; we obtained $2.23 \mathrm{eV}$ for the resonance energy, while Gianturco and Lucchese reported a value of about $1.82 \mathrm{eV}$. Again the size of the discrepancy is surprising given the nature of the calculations. Because of the low collision energy and the relatively long lifetime of the resonance, it may be necessary to go beyond the fixed-nuclei approximation to make detailed comparisons between theory and experiment. It is worth noting, in particular, that the $E_{2 u}$ resonance actually appears in the total cross section as a series of vibrational peaks extending from about $1.1 \mathrm{eV}$ to at least $1.5 \mathrm{eV} ;{ }^{10}$ the commonly cited resonance position of $1.1 \mathrm{eV}$ refers only to the approximate location of the first (and strongest) peak.

As shown in Fig. 3, at very low energies the $E_{1 u}, E_{2 g}$, and $A_{2 u}$ cross sections of Gianturco and Lucchese rise to a finite value at $0 \mathrm{eV}$, while our cross sections vanish at $0 \mathrm{eV}$. Because the $l=0$ partial wave occurs in the $A_{1 g}$ representation, one might ordinarily expect that the cross section vanish at zero energy for all other representations. ${ }^{29}$ However, benzene has a substantial quadrupole moment, ${ }^{30}$ and the resulting $1 / r^{3}$ potential will give rise to a nonzero scattering cross section at zero energy for $l>0 .{ }^{31-33}$ Indeed, using the measured quadrupole moment, ${ }^{30}$ one can estimate the magnitude of the quadrupole-induced scattering within the Born approximation $^{32}$ as about $13 \times 10^{-16} \mathrm{~cm}^{2}$, a value which appears roughly consistent with the summed results of Gianturco and Lucchese. However, it is not possible to say from the data presented in their paper whether the results of Gianturco and Lucchese are consistent with the quadrupole mechanism, which also predicts isotropic scattering and an energy-independent cross section. That the SMC cross sections do not reflect a quadrupolar contribution is probably due to the short-range nature of the trial wave function. Experimentally, Gulley et al. ${ }^{10}$ observed a rapid increase of the benzene total scattering cross section at very low energies, which they attributed to attachment promoted by the "swave leakage" mechanism of Gallup. ${ }^{34}$ Although the analysis supporting their conclusion does not take the quadrupole potential into proper account, attachment does nonetheless appear to be the most probable explanation for the observed enhancement of the total cross section.

Our differential cross sections show generally good agreement in shape with the experimental data of Gulley and Buckman, and in shape and in magnitude with the results of Gianturco and Lucchese. In the latter case, discrepancies can be found in the extreme forward direction for higher impact energies. As formation of the forward scattering peak requires high partial waves, these discrepancies are likely reflective of an insufficient representation of high partial waves in our calculation, due to the limitations of our basis set and/or the truncation of the expansion at $l=10$ in carrying out angular momentum decomposition of the scattering amplitude. It is not yet clear why the agreement in magnitude between the theoretical and experimental cross sections is better at smaller angles.

\section{SUMMARY}

We have presented results of an entirely ab initio study of low-energy electron scattering by benzene molecules us- ing the Schwinger multichannel method at both the SE and SEP levels of approximation. In general, our results agree well with previous theoretical and experimental determinations, though some puzzling areas of disagreement remain.

\section{ACKNOWLEDGMENTS}

M.H.F.B. acknowledges support from the Brazilian agency Conselho Nacional de Desenvolvimento Científico e Tecnológico (CNPq). C.W. and V.M. acknowledge support from the Department of Energy, Office of Energy Research. The authors acknowledge use of the facilities of the Center for Advanced Computing Research (CACR) at the California Institute of Technology and the valuable assistance of CACR staff, in particular Heidi Lorenz-Wirzba, Sharon Brunett, and Cris Windoffer. The authors also thank Professor S. Buckman and Dr. R. J. Gulley for provision of their results. M.H.F.B. wishes to express his gratitude to Professor V. McKoy, Dr. C. Winstead, Dr. K. Wang, and Dr. C.-H. Lee for their friendship during his visit at the California Institute of Technology.

${ }^{1}$ O. Sueoka, J. Phys. B 21, L631 (1988).

${ }^{2}$ P. Mozejko, G. Kasperski, C. Smytkowski, G. P. Karwasz, R. S. Brusa, and A. Zecca, Chem. Phys. Lett. 257, 309 (1996).

${ }^{3}$ F. Gianturco and R. R. Lucchese, J. Chem. Phys. 108, 6144 (1998).

${ }^{4}$ S. F. Wong and G. J. Schulz, Phys. Rev. Lett. 35, 1429 (1975).

${ }^{5}$ M. Ben Arfa and M. Tronc, J. Electron Spectrosc. Relat. Phenom. 50, 117 (1990).

${ }^{6}$ J. S.-Y. Chao and K. D. Jordan, J. Phys. Chem. 91, 5578 (1987).

${ }^{7}$ I. Nenner and G. J. Schulz, J. Chem. Phys. 62, 1747 (1975).

${ }^{8}$ R. Azria and G. J. Schulz, J. Chem. Phys. 62, 573 (1975).

${ }^{9}$ M. Allan, Helv. Chim Acta 65, 2009 (1982).

${ }^{10}$ R. J. Gulley, S. L. Lunt, J-P. Ziesel, and D. Field, J. Phys. B 31, 2735 (1998).

${ }^{11}$ R. J. Gulley and S. J. Buckman, J. Phys. B 32, L405 (1999).

${ }^{12}$ R. J. Gulley and S. J. Buckman, private communication (1999).

${ }^{13}$ M. H. F. Bettega, L. G. Ferreira, and M. A. P. Lima, Phys. Rev. A 47, 1111 (1993).

${ }^{14}$ M. T. do N. Varella, M. H. F. Bettega, M. A. P. Lima, and L. G. Ferreira, J. Chem. Phys. 111, 6396 (1999); M. T. do N. Varella, A. P. P. Natalense, M. H. F. Bettega, and M. A. P. Lima, Phys. Rev. A 60, 3684 (1999).

${ }^{15}$ A. P. P. Natalense, L. G. Ferreira, and M. A. P. Lima, Phys. Rev. Lett. 81, 3832 (1998).

${ }^{16}$ V. McKoy, C. Winstead, and C.-H. Lee, J. Vac. Sci. Technol. A 16, 324 (1998).

${ }^{17}$ K. Takatsuka and V. McKoy, Phys. Rev. A 24, 2473 (1981); 30, 1734 (1984).

${ }^{18}$ M. A. P. Lima, L. M. Brescansin, A. J. R. da Silva, C. Winstead, and V. McKoy, Phys. Rev. A 41, 327 (1990).

${ }^{19}$ C. Winstead and V. McKoy, Adv. At., Mol., Opt. Phys. 36, 183 (1996).

${ }^{20}$ K. H. Jordan and P. D. Burrow, Chem. Rev. 87, 557 (1987).

${ }^{21}$ K. Aflatooni, G. A. Gallup, and P. D. Burrow, J. Phys. Chem. A 102, 6205 (1998).

${ }^{22}$ G. Herzberg, Molecular Spectra and Molecular Structure, Vol. III (Van Nostrand, New York, 1966), p. 999.

${ }^{23}$ M. W. Schmidt, K. K. Baldridge, J. A. Boatz, S. T. Elbert, M. S. Gordon, J. H. Jensen, S. Koseki, N. Matsunaga, K. A. Nguyen, S. J. Su, T. L. Windus, M. Dupuis, and J. A. Montgomery, J. Comput. Chem. 14, 1347 (1993).

${ }^{24}$ C. Winstead and V. McKoy, Phys. Rev. A 57, 3589 (1998).

${ }^{25}$ C.-H. Lee, C. Winstead, and V. McKoy, J. Chem. Phys. 111, 5056 (1999).

${ }^{26}$ C. W. Bauschlicher, J. Chem. Phys. 72, 880 (1990).

${ }^{27}$ T. N. Rescigno, B. H. Lengsfield, and C. W. McCurdy, in Modern Electronic Structure Theory, edited by D. F. Yarkony (World Scientific, Singapore, 1995), Part I, p. 501. 
${ }^{28}$ S. L. Altmann, Proc. Cambridge Philos. Soc. 53, 343 (1957).

${ }^{29}$ E. P. Wigner, Phys. Rev. 73, 1002 (1948).

${ }^{30}$ M. R. Battaglia, A. D. Buckingham, and J. H. Williams, Chem. Phys. Lett. 78, 421 (1981).

${ }^{31}$ E. Gerjuoy and S. Stein, Phys. Rev. 97, 1671 (1955).
${ }^{32}$ L. Wijnberg, J. Chem. Phys. 44, 3864 (1966).

${ }^{33}$ M. A. Morrison, N. F. Lane, and L. A. Collins, Phys. Rev. A 15, 2186 (1977).

${ }^{34}$ G. A. Gallup, Phys. Rev. A 34, 2746 (1986); J. Chem. Phys. 99, 827 (1993). 